\title{
Efecto del Tratamiento con Acelerador Lineal Sobre el Flujo Salival en Pacientes con Cáncer de Cabeza y Cuello
}

\author{
Effect of Treatment with Linear Accelerator on Salivary \\ Flow in Patients with Head and Neck Cancer
}

Sarita Angelita Delgado Valladares* \& Angel Steven Asmat Abanto

DELGADO, V. S. A. \& ASMAT, A. A. S. Efecto del tratamiento con acelerador lineal sobre el flujo salival en pacientes con cáncer de cabeza y cuello. Int. J. Odontostomat., 8(2):241-245, 2014.

RESUMEN: El objetivo de este estudio fue determinar el efecto del tratamiento con acelerador lineal sobre el flujo salival en pacientes con cáncer de cabeza o cuello. El estudio presentó un diseño pre-experimental y se desarrolló en el Centro de Radioterapia Es Salud - La Esperanza (Trujillo, Perú). La muestra estuvo conformada por 20 pacientes, entre 30 y 69 años, que cumplieron los criterios de elegibilidad. El flujo salival se recolectó antes de recibir la terapia y una semana después, mediante expectoración y se evaluó por medio de la sialometría. El análisis estadístico se realizó mediante la prueba T de Student para comparación de medias, con un valor $p<0,005$. El tratamiento con acelerador lineal provocó disminución del flujo salival en pacientes con cáncer de cabeza o cuello $(p=0,000)$. En hombres, la radiación disminuye el flujo salival $(p=0,008)$; mientras que, en las mujeres el flujo salival no evidencia disminución significativa $(p=0,0636)$; asimismo, no existe diferencia con respecto a la variación de los flujos salivales entre hombres y mujeres $(p=0,122)$. El tratamiento con acelerador lineal provoca disminución del flujo salival en pacientes con cáncer de cabeza o cuello, sin diferencia por sexo.

PALABRAS CLAVE: radiación, flujo salival, cáncer de cabeza y cuello.

\section{INTRODUCCIÓN}

La saliva es una secreción compleja (Tenovuo, 1997) y juega un papel importante en la homeostasis oral (Grundmann et al., 2009). El volumen salival alcanza su máximo alrededor del mediodía y disminuye de forma considerable durante el sueño (Nauntofte et al., 2003; Dodds et al., 2005). Puede llegar hasta 1,5 litros por día, con un promedio entre $0,3-0,5 \mathrm{ml} / \mathrm{min}$ (Gomez de Ferraris \& Campos Muñoz, 2009).

La hiposalivación obstaculiza el papel protector de la saliva. Esto puede producirse por enfermedades sistémicas, estrés, algunos medicamentos (Taybos, 1998), terapia quirúrgica, alteraciones psicológicas (Fox, 1989), radiaciones en cabeza y cuello (Tschoppe et al., 2012).

Por otro lado, la radioterapia es un tratamiento no quirúrgico para el cáncer en donde se descargan rayos externos de alta energía no invasora usando un acelerador lineal, o interna, a través de implantes de semillas insertadas cerca del tumor (Perry, 2007; Raspall, 2002)

El acelerador lineal tiene la capacidad de lesionar las células cancerosas, mientras que las células normales pueden reparar este daño y sobrevivir (Perry). Sin embargo, no se pueden evitar efectos secundarios significativos en las áreas involucradas (Specht, 2002; Lopes et al., 1998). Uno de los efectos adversos de la radiación para tratar el cáncer de cabeza y cuello es una marcada disminución de la tasa de flujo salival durante la primera semana de tratamiento, la que puede ir disminuyendo aún más (Henson et al., 1999).

La pérdida de células acinares y la contracción glandular que se produce durante la fase aguda (Robar et al., 2007; Hoebers et al., 2008) afecta la com-

\footnotetext{
* Cirujano Dentista. Estudiante de la Maestría en Docencia e Investigación en Estomatología. Universidad Alas Peruanas, Trujillo, Perú.

" Maestro en Estomatología, Docente de la Escuela de Estomatología, Universidad Alas Peruanas, Trujillo, Perú.

**** Docente de Pre y Postgrado, Universidad Privada Antenor Orrego, Trujillo, Perú.
} 
posición de la saliva volviéndose densa, pegajosa y viscosa dificultando su función (Rovirosa, 2000; Suárez et al., 2007). En la fase crónica las personas continúan con disminución significativa por varios meses o años después de la radiación (Eisbuch et al., 2001; Dirix et al., 2006; Li et al., 2007).

Cuando la producción de saliva desciende y el $\mathrm{pH}$ se acidifica, los iones calcio y fósforo no pueden remineralizar el esmalte (Bascones, 2007), favoreciendo la sobreinfección, aumento en la frecuencia de caries (Rovirosa), mucositis y malnutrición (Hancock et al., 2003; Nguyen et al., 2007; Ogawara et al., 2002).

Debido que la regeneración glándular tomará tiempo, dependiendo de la dosis total de radiación (Verdú Rotellar et al., 2002; López-Jornet, 2002), se requiere un equipo de profesionales para brindar al paciente cuidados y hábitos, durante y después de haber recibido el tratamiento (Hancock et al.).

El objetivo fue determinar el efecto del tratamiento con acelerador lineal sobre el flujo salival en pacientes con cáncer de cabeza o cuello. Con esto se puede informar objetivamente a las instituciones competentes y sugerir la participación constante del odontólogo, para tratar los efectos de la hiposalivación.

\section{MATERIAL Y MÉTODO}

El presente fue un estudio pre-experimental, que se realizó en "Centro de Radioterapia Es Salud - la Esperanza (Trujillo - Perú)», entre mayo y septiembre de 2013. La población bajo estudio estuvo constituida por los pacientes que acudieron con indicación de radioterapia. El tamaño muestral fue de 20 sujetos, determinado por la fórmula que corresponde a comparación de medias en datos pareados, considerando $\mathrm{m} 1=0,500, \mathrm{~m} 2=0,271$ y s $=0,252$, valores obtenidos mediante prueba piloto.

Se eligieron para el estudio los pacientes con diagnóstico de cáncer en cabeza o cuello, entre 30 a 69 años de edad y que recibían por primera vez el tratamiento de radioterapia. Se excluyeron a los pacientes con alguna afección previa de las glándulas salivales, con enfermedad o tratamiento distinto o adicional a radioterapia y a los pacientes que no aceptaron participar del estudio. Una vez iniciado el estudio, la unidad fue eliminada si el paciente abandonaba el tratamiento.
Para la ejecución, se contó con la aprobación de la Facultad de Medicina Humana y Ciencias de la Salud de la Universidad Alas Peruanas y del Comité de Investigación y Ética de la Red Asistencial La Libertad - ESSALUD, considerando los principios de la Declaración de Helsinki y de la Ley General de Salud del Perú (Ley № 26842).

Mediante la historia clínica se seleccionaron a los pacientes que cumplían con los criterios mencionados. De aceptar, firmaron el consentimiento informado. Se solicitó a los sujetos abstenerse de comer, beber, fumar y realizar higiene oral por lo menos dos horas antes de la colección de saliva. Las muestras fueron recogidas bajo las mismas condiciones, por el investigador principal, entre las 08:00 y 10:00 horas am, con el propósito de reducir en lo posible la influencia circadiana. Posteriormente se enjuagaron la cavidad oral con agua purificada y después de dos minutos de acomodación, cada sujeto deglutió toda la saliva remanente. La saliva sin estimular fue colectada durante un periodo de cinco minutos por medio de expectoración, en un tubo desechable estéril de polipropileno de $15 \mathrm{ml}$ de capacidad.

Como terapia, todos los pacientes recibieron 200cGy de radiación por sesión durante 5 días. Después de una semana, se procedió a tomar nuevamente las muestras de saliva, siguiendo el mismo procedimiento estandarizado. Se realizó el análisis gravimétrico para determinar los promedios de flujo salival. Los promedios de secreción se expresaron en $\mathrm{ml} / \mathrm{min}$, después de los cinco minutos de colección (considerando que $1,0 \mathrm{~g}=1,0 \mathrm{ml}$ ).

Los datos fueron procesados y presentados en tablas con medias y desviación estándar. El efecto de la radiación sobre el flujo salival se evaluó empleando la prueba T de Student para comparación de medias de datos pareados, y al comparar la variación del flujo salival entre hombres y mujeres para medias en grupos independientes. La significancia estadística considerada fue al $5 \%$.

\section{RESULTADOS}

Se pudo evaluar un total de 20 pacientes (11 hombres y 9 mujeres), entre 30 y 69 años de edad. La radiación produjo disminución del flujo salival en pacientes adultos con cáncer de cabeza o cuello sometidos a tratamiento mediante acelerador lineal $(p=0,000)$ (Tabla I). 
Tabla I. Efecto de la radiación sobre el flujo salival en pacientes adultos con cáncer de cabeza o cuello sometidos a tratamiento mediante acelerador lineal.

\begin{tabular}{lccc}
\hline & \multicolumn{3}{c}{ Flujo salival $(\mathrm{ml})$} \\
\cline { 2 - 4 } & Antes & Después & Variación \\
\hline Media & 0,490 & 0,260 & 0,230 \\
D. Estándar & 0,107 & 0,182 & 0,230 \\
Prueba T & - & - & 4,479 \\
p & - & - & 0,000 \\
\hline
\end{tabular}

En hombres, la radiación disminuyó el flujo salival $(p=0,008)$; mientras que en las mujeres el flujo salival no evidencia disminución significativa $(p=0,0636)$; asimismo, no existe diferencia con respecto a la variación de los flujos salivales entre hombres y mujeres $(p=0,122)$ (Tabla II).

Tabla II. Flujo salival después de la radiación en pacientes adultos con cáncer de cabeza o cuello sometidos a tratamiento mediante acelerador lineal en el Centro de Radioterapia de la Esperanza Es Salud- Trujillo, 2013, según sexo.

\begin{tabular}{lcccccc}
\hline & \multicolumn{3}{c}{ Hombres } & \multicolumn{3}{c}{ Mujeres } \\
\cline { 2 - 7 } & Antes & Después & Variación & Antes & Después & Variación \\
\hline Media & 5,500 & 2,400 & 3,100 & 4,300 & 2,800 & 1,500 \\
D. Estándar & 0,850 & 1,430 & 1,853 & 0,949 & 2,201 & 2,506 \\
Prueba T & 2,979 & $-0,482$ & 1,624 & - & - & - \\
p & 0,008 & 0,636 & 0,122 & - & - & - \\
\hline
\end{tabular}

\section{DISCUSIÓN}

El flujo salival resulta importante en el proceso de homeostasis oral y contribuye como protector ante la presencia de factores fisiológicos o factores externos (Zarate et al., 2004; Navazesh \& ADA Council on Scientific Affairs and Division of Science, 2003).

En la población evaluada, el flujo salival promedio, antes de la radiación fue de $0,49 \mathrm{ml}$, el que se encuentra dentro de los parámetros normales. Después de la radiación, el flujo salival disminuyó significativamente, como promedio a $0,26 \mathrm{ml}$. Este hallazgo es similar al reportado por Henson et al.

En estos pacientes, es necesario brindar la atención debida para prevenir alteraciones en las estructuras bucales, como el aumento del índice de caries, enfermedad periodontal e inflamación de las mucosas, que dificultan la alimentación, disminuyendo las defensas del paciente (Hancook et al.; Nguyen et al.; Ogawara et al.).

Se encontró, una variación significativa después de la radiación en los hombres, probablemente debido a que él hay un mayor promedio de flujo salival basal comparado con la mujer.

Una limitación importante del presente estudio es su diseño pre-experimental, donde no existe grupo control. Debido a la naturaleza de la enfermedad, no se puede comparar con pacientes que reciban dosis de radiación distintas y poder evidenciar diferencias que aporten más información al estudio.

El motivo de este diseño fue porque en el Centro de Radioterapia se aplica la misma dosis de radiación a todos los pacientes con cáncer de cabeza o cuello, la que es establecida por la especialista a cargo del tratamiento. Para los investigadores no es factible indicar la dosis, respetando en todo momento las consideraciones éticas, que indican priorizar el beneficio en la salud para el sujeto investigado.

Concluimos que la radiación produce disminución del flujo salival en pacientes adultos de ambos sexos con cáncer de cabeza o cuello sometidos a tratamiento mediante acelerador lineal, siendo significativa sólo en los hombres; asimismo, no existe diferencia con respecto a la variación de los flujos salivales entre hombres y mujeres.

Se sugiere que la atención de los pacientes sea desde un enfoque multidisciplinario, donde el odontólogo juegue un papel importante para tratar y prevenir las afecciones orales derivadas de este efecto secundario de la terapia de cáncer de cabeza y cuello.

AGRADECIMIENTOS. A la Dra. Maria Alejandra Arens Benítez, Coordinadora del Centro de Radioterapia del Hospital Víctor Lazarte Echegaray, y a los pacientes evaluados por su desinteresada colaboración en el desarrollo del presente trabajo. 
DELGADO, V. S. A. \& ASMAT, A. A. S. Effect of treatment with linear accelerator on salivary flow in patients with head and neck cancer. Int. J. Odontostomat., 8(2):241-245, 2014.

ABSTRACT: The objective of the present study was to determine the effect of treatment with linear accelerator on salivary flow in patients with head and neck cancer. The study had a pre-experimental design and was developed at the Radiotherapy Centre Es Salud La Esperanza (Trujillo, Peru). The sample consisted of 20 patients, between 30 and 69 years, who met the eligibility criteria. Salivary flow was collected before receiving therapy and a week later, by expectoration and evaluated through sialometry. Statistical analysis was performed using Student 's t test for comparison of means with $p$ $<0.005$. Linear accelerator treatment decreased salivary flow in patients with head and neck cancer $(p=0.000)$. In men, the radiation decreases salivary flow $(p=0.008)$, while in women there was no evidence of significant salivary flow decrease $(p=0.0636)$, also, there is no difference with respect to variation in salivary flow between men and women $(p=0.122)$. Linear accelerator treatment decreased salivary flow in patients with head and neck cancer, without sex differences.

KEY WORDS: radiation, salivary flow, head and neck cancer.

\section{REFERENCIAS BIBLIOGRÁFICAS}

Bascones, A.; Tenovuo, J; Ship, J.; Turner, M.; Mac-Veigh, I.; López-Ibor, J. M.; Albi, M.; Lanzós, E. \& Aliaga, A. Conclusiones del Simposium 2007 de la Sociedad Española de Medicina Oral sobre "Xerostomía. Síndrome de Boca Seca. Boca Ardiente". Av. Odontoestomatol., 23(3):119-26, 2007.

Dirix, P.; Nuyts, S. \& Van den Bogaert, W. Radiation-induced xerostomia in patients with head and neck cancer: a literature review. Cancer, 107(11):2525-34, 2006.

Dodds, M. W.; Johnson, D. A. \& Yeh, C. K. Health benefits of saliva: a review. J. Dent., 33(3):223-33, 2005.

Eisbruch, A; Kim, H. M.; Terrell, J. E.; Marsh, L. H.; Dawson, L. A. \& Ship, J. A. Xerostomia and its predictors following parotid-sparing irradiation of head-and-neck cancer. Int. J. Radiat. Oncol. Biol. Phys., 50(3):695-704, 2001.

Fox, P. C. Saliva composition and its importance in dental health. Compend. Suppl., 13(2):457-60, 1989.

Gomez de Ferraris, M. E. \& Campos Muñoz, A. Histología, Embriología e Ingeniería Tisular Bucodental. $3^{a}$ ed. Madrid, Médica Panamericana, 2009.

Grundmann, O.; Mitchell, G. C. \& Limesand, K. H. Sensitivity of salivary glands to radiation: from animal models to therapies. J. Dent. Res., 88(10):894-903, 2009.

Hancock, P. J.; Epstein, J. B. \& Sadler, G. R. Oral and dental management related to radiation therapy for head and neck cancer. J. Can. Dent. Assoc., 69(9):585-90, 2003.

Henson, B. S.; Eisbruch, A.; D'Hondt, E. \& Ship, J. A. Twoyear longitudinal study of parotid salivary flow rates in head and neck cancer patients receiving unilateral neck parotid-sparing radiotherapy treatment. Oral Oncol., 35(3):234-41, 1999.
Hoebers, F. J.; Kartachova, M.; de Bois, J.; van den Brekel, M. W.; van Tinteren, H.; van Herk, M.; Rasch, C. R.; Valdés Olmos, R. A. \& Verheij, M. 99mTc Hynic-rhAnnexin $V$ scintigraphy for in vivo imaging of apoptosis in patients with head and neck cancer treated with chemoradiotherapy. Eur. J. Nucl. Med. Mol. Imaging, 35(3):509-18, 2008.

Li, Y.; Taylor, J. M.; Ten Haken, R. K. \& Eisbruch, A. The impact of dose on parotid salivary recovery in head and neck cancer patients treated with radiation therapy. Int. J. Radiat. Oncol. Biol. Phys., 67(3):660-9, 2007.

Lopes, M. A.; Coletta, R. D.; Alves, F. A.; Abbade, N. \& Rossi, J.R. A. Reconhecendo e controlando os efeitos colaterais da radioterapia. Rev. Assoc. Paul. Cir. Dent., 52(3):2414, 1998.

López-Jornet, P. Alteraciones de las glándulas salivales. Murcia, Universidad de Murcia, 2002.

Nauntofte, B.; Tenevuo, J. O. \& Lagerlöf, F. Secretion and composition of saliva. In: Fejerskov, O. \& Kidd, E. (Eds.). Dental Caries: The disease and its clinical management. Oxford, Blackwell Munksgard, 2003.

Navazesh, M. \& ADA Council on Scientific Affairs and Division of Science. How can oral health care providers determine if patients have dry mouth? J. Am. Dent. Assoc., 134(5):613-20, 2003.

Nguyen, N. P.; Vos, P.; Karlsson, U.; Nguyen, P.; Dutta, S.; Lemanski, C.; Ludin, A.; Rose, S.; Nguyen, L. M.; Ward, H.; Huang, S. \& Sallah, S. Quality of life following chemoradiation and postoperative radiation for locally advanced head and neck cancer. O.R.L. J. Otorhinolaryngol. Relat. Spec., 69(5):271-6, 2007.

Ogawara, Y.; Kishishita, S.; Obata, T.; Isazawa, Y.; Suzuki, 
DELGADO, V. S. A. \& ASMAT, A. A. S. Efecto del tratamiento con acelerador lineal sobre el flujo salival en pacientes con cáncer de cabeza y cuello. Int. J. Odontostomat., 8(2):241-245, 2014.

T.; Tanaka, K.; Masuyama, N. \& Gotoh, Y. Akt enhances Mdm2-mediated ubiquitination and degradation of p53. J. Biol. Chem., 277(24):21843-50, 2002.

Perry, M. Principles of cancer therapy. $23^{\text {th }}$ ed. Philadelphia, Elsevier, 2007

Raspall, G. Cirugía maxilofacial: patología quirúrgica de la cara, boca, cabeza y cuello. Madrid, Médica Panamericana, 2002

Robar, J. L.; Day, A.; Clancey, J.; Kelly, R.; Yewondwossen, M.; Hollenhorst, H.; Rajaraman, M. \& Wilke, D. Spatial and dosimetric variability of organs at risk in head-andneck intensity-modulated radiotherapy. Int. J. Radiat. Oncol. Biol. Phys., 68(4):1121-30, 2007.

Rovirosa, A. Xerostomía radioinducida. Barcelona, Hospital Clinic i Universitari, 2000.

Suárez, C.; Gil-Carcedo, L.; Marco, C.; Medina, J.; Ortega, P. \& Trinidad, J. Tratado de otorrinolaringología y cirugía de cabeza y cuello. $2^{a}$ ed. Madrid, Médica Panamericana, 2007.

Specht, L. Oral complications in the head and neck radiation patient. Introduction and scope of the problem. Support Care Cancer, 10(1):36-9, 2002.

Taybos, G. M. Xerostomia. Common patient complaint and challenging dental management problem. Miss. Dent. Assoc. J., 54(3):24-5, 1998.

Tenovuo, J. Salivary parameters of relevance for assessing caries activity in individuals and populations. Community Dent. Oral. Epidemiol., 25(1):82-6, 1997.

Tschoppe, P.; Wolgin, M.; Pischon, N. \& Kielbasa, A. M. Factores etiológicos de la hiposalivación y sus consecuencias en la salud oral. Quintessence, 25(1):41-52, 2012.

Verdú Rotellar, J. M.; Algara López, M.; Foro Arnalot, P.; Domínguez Tarragona, M. \& Blanch Mon, A. Atención a los efectos secundarios de la radioterapia. Medifam., 12(7):16-33, 2002.

Zarate, D. A. N.; Leyva, H. E. R. \& Martínez, M. F. Determinación de $\mathrm{pH}$ y proteínas totales en saliva en pacientes con y sin aparatología ortodóncica fija (estudio piloto). Rev. Odontol. Mex., 8(3):59-63, 2004.
Dirección para Correspondencia:

C.D. Ms. Angel Steven Asmat Abanto

Escuela de Estomatología

Universidad Alas Peruanas

Trujillo

PERÚ

Email: aasmata1@upao.edu.pe

Recibido : 03-12-2013

Aceptado: 03-02-2014 